\title{
Scanning Tunneling Microscopy Fractal Characterization of Poly(o-toluidine) Films Produced Electrochemically on Polyfaceted Gold Single Crystal Spheres
}

\author{
P. Ocón, P. Herrasti, and J. M. Vara \\ Departamento de Quimica Fisica Aplicada C-II, Universidad Autónoma de Madrid 28049, Madrid, Spain
}

L. Vázquez

Instituto de Ciencia de Materiales, CSIC, Departamento de Aplicada, C-XII, Universidad Autónoma de Madrid (UAM), 28049, Madrid, Spain

\author{
R. C. Salvarezza and A. J. Arvia* \\ Instituto de Investigaciones Fisicoquimicas Teóricas y Aplicadas (INIFTA), Sucursal 4 , \\ Casilla de Correo 16, (1900) La Plata, Argentina
}

Received: September 10, 1993; In Final Form: December 9, $1993^{\circ}$

\begin{abstract}
The topography evolution of a poly(o-toluidine) layer (the object) electrodeposited on a polyfaceted Au single crystal from $0.5 \mathrm{M} \mathrm{H}_{2} \mathrm{SO}_{4}+0.65 \mathrm{Mo}$-toluidine aqueous solution at $25^{\circ} \mathrm{C}$ has been studied combining nanometer scale ex-situ scanning tunneling microscopy and electrochemical techniques. A thin polymer layer structure consists of nodular elements following the substrate topography, whereas a thick polymer structure corresponds to a rather disordered structure made up of agglomerates formed by nodular elements with average diameter size $d_{\mathrm{s}} \cong 15 \mathrm{~nm}$. In contrast to the former one, this structure is independent of the substrate topography. For thick layers $\xi$, the root-mean-square roughness of the polymer coating, and $L_{\mathrm{s}}$, the scan length, fulfill a $\xi \propto L^{\alpha}$ relationship with $\alpha=0.33 \pm 0.05$ for $L_{\mathrm{s}}>d_{\mathrm{s}}$ and $\alpha=0.85 \pm 0.05$ for $L_{\mathrm{s}}<d_{\mathrm{s}}$. Accordingly, the polymer layer surface behaves as a self-affine fractal. For $L_{\mathrm{s}}>d_{\mathrm{s}}$ the value of $\alpha$ agrees with that predicted for moving interfaces generated by the Eden model, whereas for $L_{\mathrm{s}}<d_{\mathrm{s}}$ the value of $\alpha$ approaches that expected from an Euclidean surface. The topographic analysis of these polymer layers can be related to the kinetics of electrochemical reactions at polymer-coated $\mathrm{Au}$ electrodes.
\end{abstract}

\section{Introduction}

Since earlier works on conducting polymer-coated electrodes, ${ }^{1-4}$ an increasing number of investigations have been devoted to the surface and the structural properties, ${ }^{5-7}$ the conduction models, ${ }^{8}$ and growth mechanisms for these materials. ${ }^{9}$ At present, there is considerable interest in these topics as a number of conducting polymers are useful as supporting materials for metal catalysts, electrode material for electrochemical energy conversion, ion sensors, organic semiconductors, color displays, and optoelectronic devices. Aromatic conducting polymers such as polyaniline exhibit good Coulombic reversibility in interconversion between the conductive and insulating state and have been applied to charge storage in secondary batteries.

Recent scanning tunneling microscopy (STM) and atomic force microscopy (AFM) imaging of conducting polymers has furnished relevant data about the structure of thick polymer layers electrodeposited on well-defined crystalline metal and carbon substrates. ${ }^{5-7}$ In the case of electropolymerized polypyrrole and polythiophene the polymer structure changes with film thickness. 5,7 Thus, in the 20-90-nm film thickness range a fibular structure which turns into a nodular polymer structure with increasing film thickness has been observed. 5,7 As the film becomes rougher, the degree of surface disorder increases, and then the film topography resembles that observed for fastly grown thin metal films. ${ }^{10}$ In this case topographic characterization allowed us to link the degree of surface disorder with the surface growth mechanism and the kinetics of electrochemical reactions on these materials. ${ }^{10-12}$ Previous voltammetric studies related to the formation of polyaniline ${ }^{9}$ and poly (2,5-dimethylaniline) deposits ${ }^{13}$ on different substrates have suggested that the structures of these

- To whom correspondence should be addressed.

- Abstract published in Advance ACS Abstracts, February 1, 1994. polymers behave as fractals.9,13 Precisely, the fractal geometry approach provides, in this case, the possibility of attempting a quantitative characterization of the degree of surface disorder at these materials. ${ }^{10}$

This paper examines the surface characteristics of conducting poly(o-toluidine) layers (polymer coating) grown electrochemically on polyfaceted Au single crystal spheres through the analysis of ex-situ STM images. The topography of these layers can be described as a rough surface resulting from the agglomeration of nodular elements ( $15 \mathrm{~nm}$ in average diameter size). By using the dynamic scaling-STM method, ${ }^{10,14}$ two different regimes can be observed. Thus, for scaling lengths smaller than the average nodular size the surface appears highly correlated, and it approaches the characteristics of an Euclidean surface. Otherwise, for scale lengths greater than the average nodular size, the surface of agglomerates resulting from the aggregation of nodular elements behaves as a self-affine fractal surface. This topographic analysis can explain the kinetics of electrochemical reactions at and inside polymer-coated $\mathrm{Au}$ electrodes in acid solutions.

\section{Experimental Section}

Poly ( $o$-toluidine) coatings were formed on polyfaceted single crystal Au spheres $\left(0.2-\mathrm{cm}^{2}\right.$ area, working electrode) from $0.5 \mathrm{M}$ $\mathrm{H}_{2} \mathrm{SO}_{4}+0.65 \mathrm{Mo}$-toluidine aqueous solution (solution I). The Au spheres were prepared by melting the tip of a 0.5 -mm-diameter Au wire with a small oxygen gas flame torch. ${ }^{15}$ The working electrode was mounted on a conventional glass-made electrochemical cell together with a $\mathrm{Pt}$ counter electrode and a $\mathrm{Hg}$ / $\mathrm{Hg}_{2} \mathrm{SO}_{4} / 0.5 \mathrm{M} \mathrm{H}_{2} \mathrm{SO}_{4}$ reference electrode. Polymer coatings were grown either at constant potential, $E_{\mathrm{g}}\left(0.20 \mathrm{~V}<E_{\mathrm{g}}<0.60\right.$ $\mathrm{V}$ ), or by repetitive triangular potential scanning at $v=0.05 \mathrm{~V}$ 


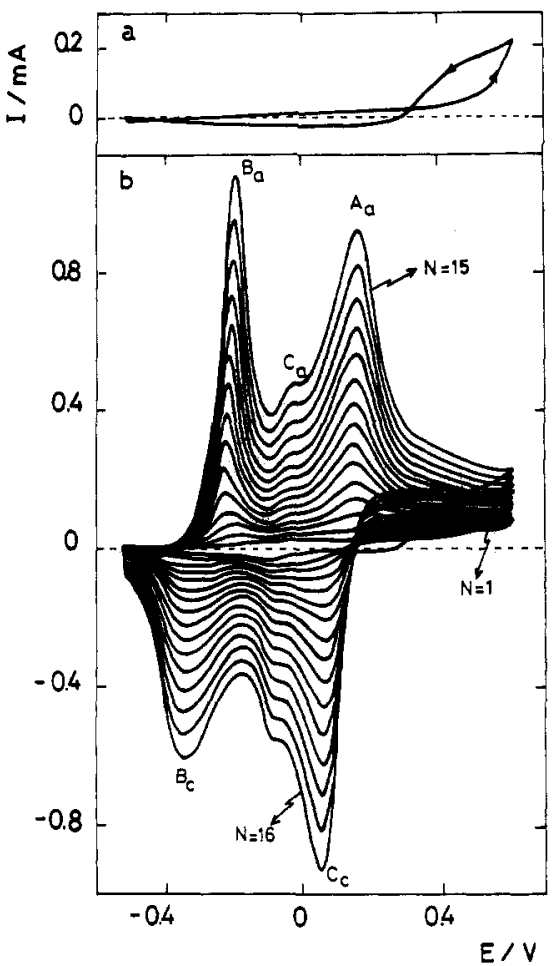

Figure 1. Voltammograms recorded at $v=0.05 \mathrm{~V} \mathrm{~s}^{-1}$ between -0.4 and $0.6 \mathrm{~V}$ in aqueous $0.5 \mathrm{M} \mathrm{H}_{2} \mathrm{SO}_{4}+0.65 \mathrm{M}$ o-toluidine solution: (a) $N=$ 1; (b) $0<N<16$. $T=25^{\circ} \mathrm{C}$.

$\mathrm{s}^{-1}$ between -0.40 and $0.60 \mathrm{~V}$. A detailed description of the polymer-coated $\mathrm{Au}$ electrode preparation has been given elsewhere. ${ }^{16}$

After polymer electrodeposition, the working electrode was removed from the electrochemical cell, carefully rinsed with deoxygenated water, and then dried under $\mathrm{N}_{2}$ at room temperature. Immediately afterward, it was placed into the sample holder of a piezotube STM with a Pt tip operating in the atmosphere.

Ex-situ STM images were obtained within a short time range and continued for no later than $6 \mathrm{~h}$ after sample removal from the cell. Under these experimental conditions no important surface contamination can be detected in the STM images.

Images were taken at a bias voltage of $0.1 \mathrm{~V}$ and 1-2 nA constant current. The piezotube was calibrated by imaging a highly oriented pyrolitic graphite surface. Pt tips were made by cutting $\mathrm{Pt}$ wires (0.5-mm diameter). STM data were acquired at a fully automated workstation and stored as digitized images with $256 \times 256$ pixels.

Occasionally, complementary information for the characterization of polymeric coatings was derived from scanning electron microscopy (SEM) and X-ray diffractometry.

The ferro-ferricyanide redox reaction (test reaction) was run at polymer-coated Au electrodes immersed in aqueous $1 \mathrm{M} \mathrm{H}_{2-}$ $\mathrm{SO}_{4}+0.01 \mathrm{M} \mathrm{NaNO}{ }_{3}+0.005 \mathrm{M} \mathrm{K}_{3}\left[\mathrm{Fe}(\mathrm{CN})_{6}\right]+0.005 \mathrm{M}$ $\mathrm{K}_{4}\left[\mathrm{Fe}(\mathrm{CN})_{6}\right]$ (solution II). Potentiostatic current transients for the anodic reaction were recorded by setting the potential in the diffusion limiting current potential range.

All solutions were prepared from ar chemicals and Milli-Q water. Prior to each experiment solutions were dearated by bubbling purified $\mathrm{N}_{2}$. Electrochemical runs were performed under a $\mathrm{N}_{2}$ atmosphere at $25^{\circ} \mathrm{C}$.

\section{Results}

3.1. Voltammetric Data. Polymer coating formation can be followed through cyclic voltammetry starting with the working electrode immersed in solution I from -0.4 to $0.6 \mathrm{~V}$ at $v=0.05$ $\mathrm{V} \mathrm{s}^{-1}$ (Figure 1a). As the potential exceeds $0.17 \mathrm{~V}$, an increase

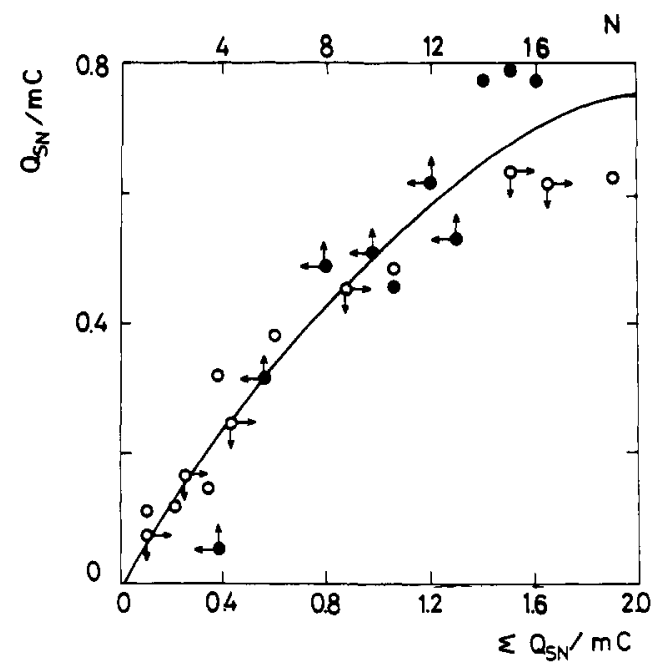

Figure 2. $Q_{\mathrm{s} N}$ vs $N$ plot (०) and (0) $Q_{\mathrm{s} N}$ vs $\Sigma_{N} Q_{\mathrm{s} N}$ plot.

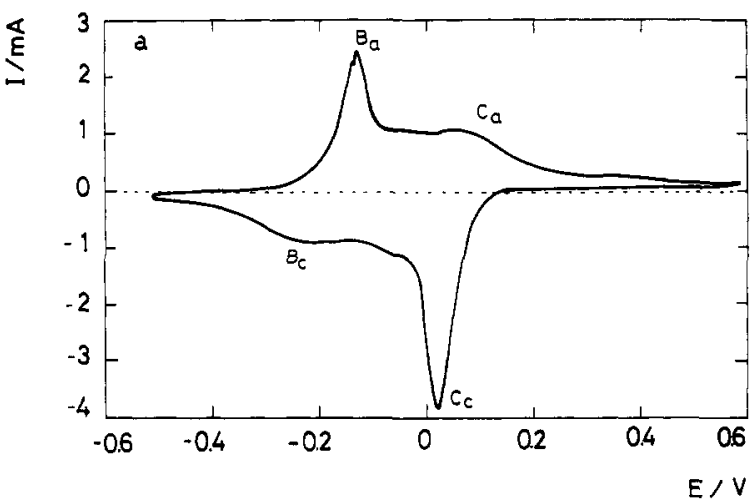

Figure 3. Voltammograms recorded at $v=0.05 \mathrm{~V} \mathrm{~s}^{-1}$ between -0.4 and $0.6 \mathrm{~V}$ for a polymer coated $\left(\sum Q_{S N}=5 \mathrm{mC}\right)$ in aqueous $0.5 \mathrm{M} \mathrm{H}_{2} \mathrm{SO}_{4}$. $T=25^{\circ} \mathrm{C}$.

in the anodic current followed by a current plateau which extends up to $0.5 \mathrm{~V}$ and then a further current increase can be observed. The reverse potential scan displays an anodic hysteresis loop which resembles those usually found in electrochemical reactions involving the nucleation and growth of a new phase. Subsequently, the potential cycling produces the change of the anodic current plateau into a well-defined current peak $A_{\mathrm{a}}$. The height of peak $\mathrm{A}_{\mathrm{a}}$ increases with $N$, the number of cycles (Figure $1 \mathrm{~b}$ ). These changes coincide with the appearance of the anodic peak $B_{a}$ at $-0.19 \mathrm{~V}$, the hump $C_{a}$ at $0.1 \mathrm{~V}$, and the cathodic peaks $B_{c}$ and $C_{c}$ at -0.34 and $0.0 \mathrm{~V}$, respectively. The anodic current recorded at a potential greater than $0.17 \mathrm{~V}$ is due to the oxidation of o-toluidine monomers yielding poly(o-toluidine).

The value of $Q_{\mathrm{SN}}$, the net polymer electrodeposition charge in the $N$ th voltammetric cycle, is given by

$$
Q_{\mathrm{S} N}=Q_{\mathrm{a} N}-Q_{\mathrm{c} N}
$$

where $Q_{\mathrm{a} N}$ and $Q_{\mathrm{cN}}$ are the overall anodic and cathodic charges involved in the $N$ th voltammetric cycle, respectively. Thus, the total charge related to polymer accumulation after $N$ cycles can be defined as $\sum Q_{\mathrm{SN}}$. The value of $Q_{\mathrm{S} N}$ increases with both $N$ and $\sum Q_{\mathrm{S} N}$ following a power law up to $\sum Q_{\mathrm{SN}}>1.60 \mathrm{mC}$ (Figure 2), a fact which suggests the development of an electrochemically active surface which, at early stages of growth, increases markedly with the polymer mass and then approaches a saturation region.

Todiscriminate the origin of the different current peaks (Figure 1a), voltammograms of polymer-coated electrodes in aqueous 1 $\mathrm{M} \mathrm{H}_{2} \mathrm{SO}_{4}$ were run between -0.5 and $0.6 \mathrm{~V}$ at $v=0.05 \mathrm{~V} \mathrm{~s}^{-1}$ (Figure 3). These voltammograms show the appearance of only peaks $B_{a}, B_{c}, C_{a}$, and hump $C_{c}$, which comprise an anodic-tocathodic charge ratio equal to 1 . Obviously, in $1 \mathrm{M} \mathrm{H}_{2} \mathrm{SO}_{4}$ peak 

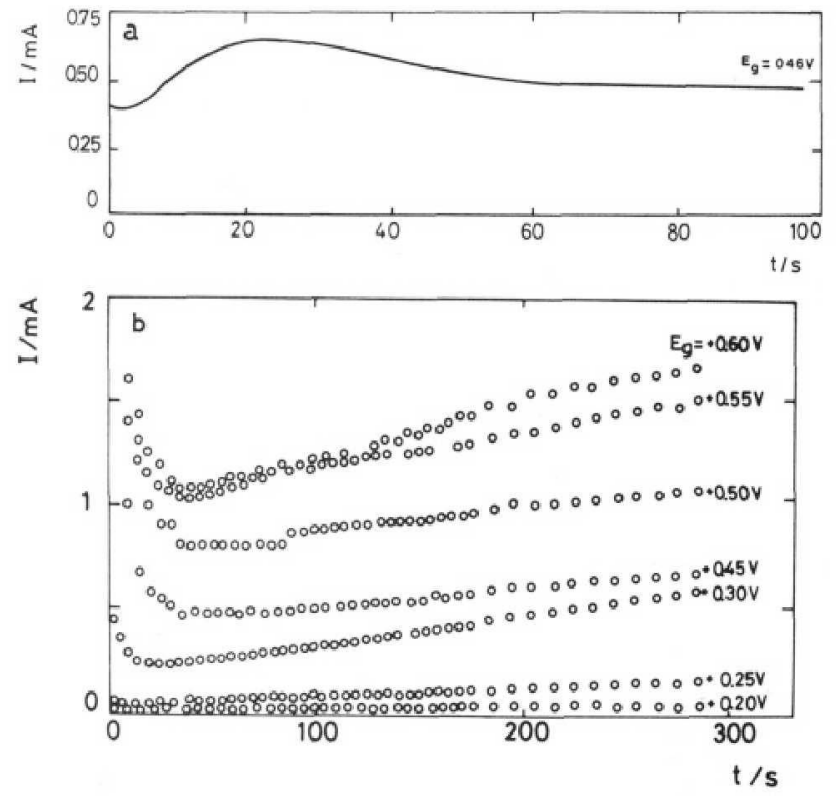

Figure 4. Current transients recorded at different $E_{\mathrm{g}}$. Au working electrode in contact with aqueous $0.5 \mathrm{M} \mathrm{H}_{2} \mathrm{SO}_{4}+0.65 \mathrm{M}$ o-toluidine solution at $t=0$. The potential was stepped from $-0.4 \mathrm{~V}$ to $E_{\mathrm{g}}(0.2 \mathrm{~V}$ $<E_{\mathrm{g}}<0.6 \mathrm{~V}$ ) at $25^{\circ} \mathrm{C}$. (a) Short- and (b) long-time range.

$\mathrm{A}_{\mathrm{a}}$, which is associated with monomer oxidation and polymer growth, cannot be observed. The equality of anodic and cathodic voltammetric charges indicates that peaks $B_{a}, B_{c}, C_{a}$, and hump $\mathrm{C}_{\mathrm{c}}$ correspond to redox reactions at active sites in the polymeric coating. As is already known for polyaniline coatings, ${ }^{9}$ these processes take place within a potential range which is significantly more negative than that related to the own monomer oxidation (peak $\mathrm{A}_{\mathrm{a}}$ ). The shapes of peaks $\mathrm{B}_{\mathrm{a}}$ and $\mathrm{C}_{\mathrm{a}}$, which exhibit a complex dependence on $v$, a re related to the electrooxidation of the polymer. This reaction can be described as a change from a conducting to a poorly conducting phase inside the polymer. ${ }^{8}$

The cyclovoltammograms of polymer-coated electrodes run in $1 \mathrm{M} \mathrm{H}_{2} \mathrm{SO}_{4}$ exhibit a sharp peak followed by a broad current plateau in both the positive and the negative potential going scans. This behavior suggests that the electrochemical reactions at polymer-coated electrodes involve two consecutive stages with their own kinetics. The same conclusion can be drawn from the change in $R$, the electric resistance of the polymer coating, with $E$, the applied potential. ${ }^{16}$ It can be seen that sharp peaks $\mathrm{B}_{\mathrm{a}}$ and $\mathrm{C}_{\mathrm{c}}$ are in the potential range where the minimum value of $R$ is found, in contrast to broad peaks $B_{c}$ and $C_{a}$. These results are consistent with electrochemical reactions involving the conversion between the conductive to the poorly conducting state of the polymer. ${ }^{8,16}$

3.2. Potentiostatic Current Transients. Potentiostatic current transients of working electrodes immersed in solution I can provide relevant information about the coating growth kinetics. These transients were recorded by stepping the potential from $-0.4 \mathrm{~V}$ to $E_{\mathrm{g}}\left(0.2 \mathrm{~V}<E_{\mathrm{g}}<0.6 \mathrm{~V}\right)$. They display an initial current decrease followed by a practically linear current increase (Figure 4). Occasionally, a current maximum can be detected in the short time range preceding the continuous current increase. This current maximum can be assigned to the initial formation of a thin and adherent polymer layer on the Au substrate, whereas the linear current increase can be related to the growth of the bulk polymer layer. ${ }^{9}$ The complex behavior of these current transients can be assigned to different stages in polymer coating formation on $\mathrm{Au}$, including nucleation and growth processes. ${ }^{9,17}$ This is consistent with the hysteresis loop found in the first voltammogram (Figure 1a).

3.3. STM Imaging. STM images focused at the [111] pole region of polyfaceted Au single crystal spheres show terraces

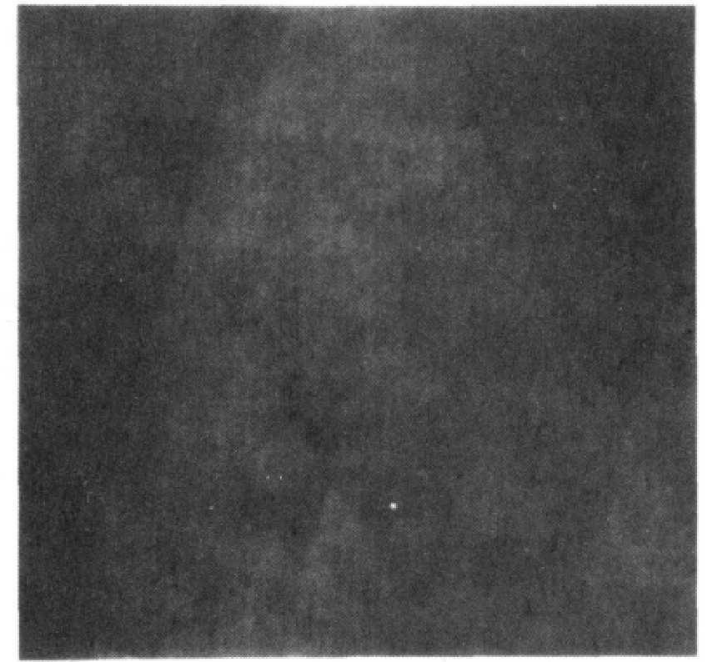

Figure 5. STM image (top view $800 \times 800 \mathrm{~nm}^{2}$ ) of a [111] pole of the polyfaceted Au single crystal substrate.

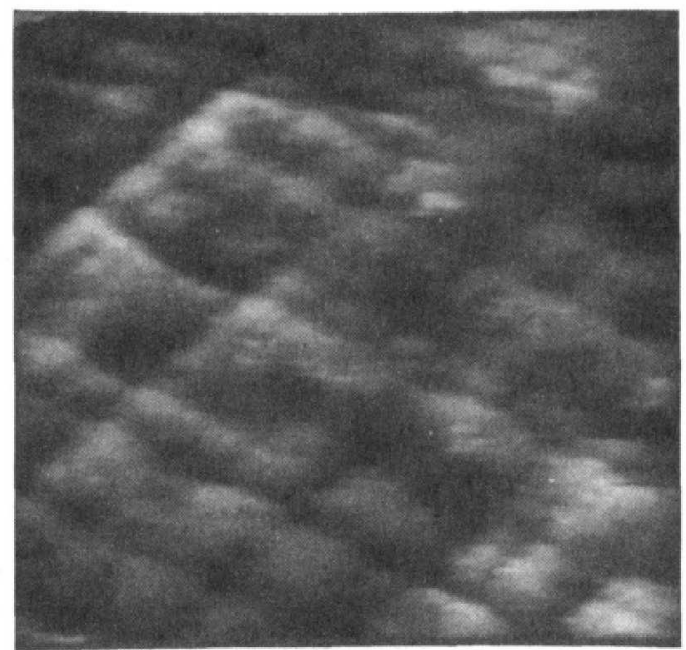

Figure 6. STM image (top view $37 \times 37 \mathrm{~nm}^{2}$ ) of a polymer-coated Au electrode $\left(Q_{\mathrm{aN}}=0.553 \mathrm{C}, \boldsymbol{E}_{\mathrm{g}}=0.35 \mathrm{~V}\right)$.

with monoatomic steps, the step directions forming $60^{\circ}$ angles (Figure 5), in agreement with previous results. ${ }^{18}$

STM images of a relatively thin polymer coating $\left(Q_{\mathrm{aN}}=0.553\right.$ $\mathrm{C}, E_{\mathrm{g}}=0.35 \mathrm{~V}$ ) (Figure 6) show polymer chains formed by nodular elements. In these cases, nodular elements which form the polymer structure are linearly ordered and reflect the $60^{\circ}$ angle direction which is typical of the Au substrate topography (Figure 5). Therefore, it can be concluded that there is a clear influence of the Au substrate on the structure of polymer coatings at the early stages of growth.

Otherwise, STM images of relatively thick polymer-coated $\left(Q_{\mathrm{a} N}=22 \mathrm{C}, E_{\mathrm{g}}=0.35 \mathrm{~V}\right)$ electrodes (Figure 7) show that the influence of the Au substrate topography on the polymer coating structure has disappeared. Polymer coating domains ranging from $1000 \times 1000$ to $50 \times 50 \mathrm{~nm}^{2}$ (Figure 7A) display disordered and rough surface structures consisting of nodular elements and branched voids at all magnifications; i.e., at those length scales the polymer coating surface behaves as a fractal. The minimum nodular element size determined from STM imaging is about 5 $\mathrm{nm}$. The nodular structure is similar to that reported for electropolymerized polypyrrole and polythiophene by STM imaging. ${ }^{5,7}$ High-resolution STM imaging attempting to resolve the fine structure of poly( $o$-toluidine) nodules was unsuccessful. Similar results have been recently reported for polyaniline and polyaniline-based materials. ${ }^{19}$ Occasionally, crystalline regions can also be seen at short length scales (Figure 7B).

3.4. X-ray Diffractometry. The X-ray diffractograms of 


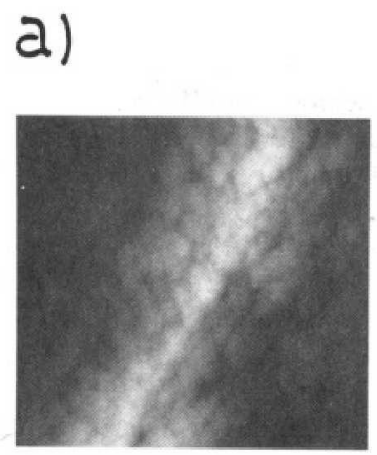

C)

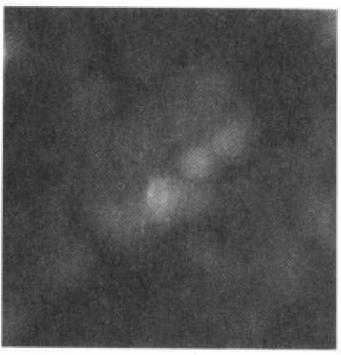

b)

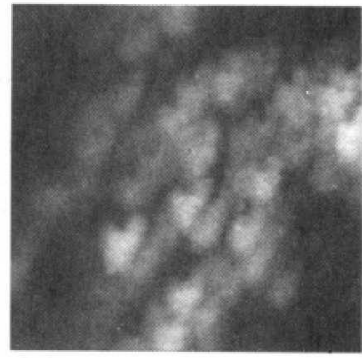

d)
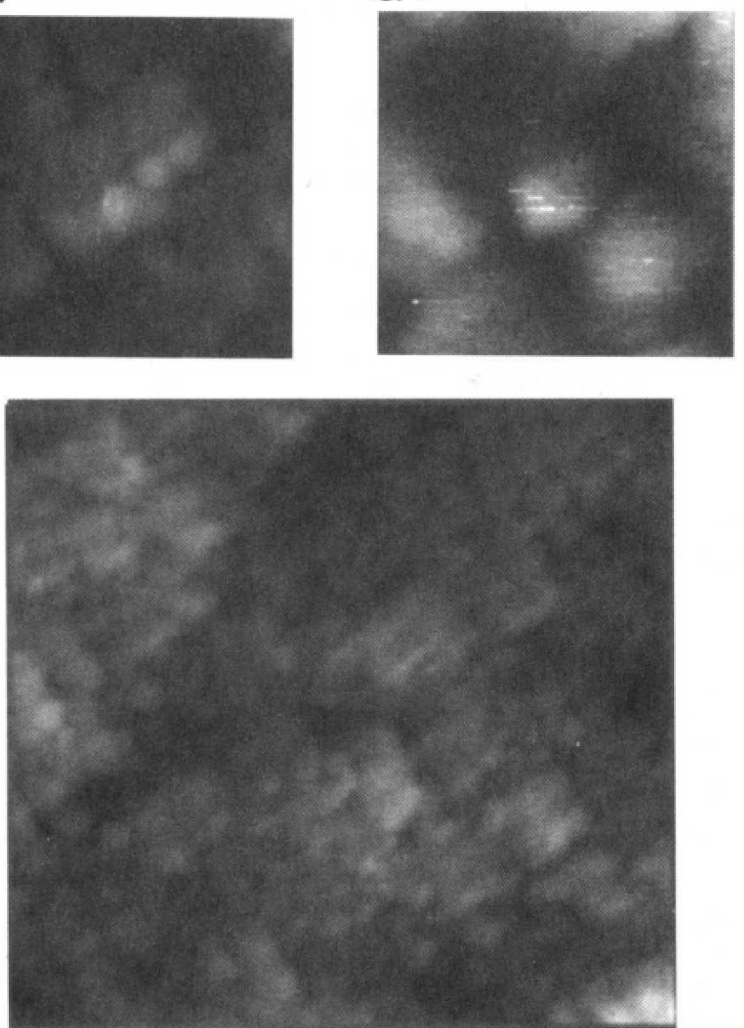

Figure 7. STM images (top view) at different magnifications of a polymercoated Au electrode $\left(Q_{\mathrm{aN}}=22 \mathrm{C}, E_{\mathrm{g}}=0.35 \mathrm{~V}\right)$. A (top): (a) $970 \times$ $970 \mathrm{~nm}^{2}$, (b) $280 \times 280 \mathrm{~nm}^{2}$, (c) $80 \times 80 \mathrm{~nm}^{2}$, (d) $50 \times 50 \mathrm{~nm}^{2}$. B (bottom): $300 \times 300 \mathrm{~nm}^{2}$; a certain degree of crystallinity can be observed in this image.

polymer coatings (Figure 8 ) display peaks which correspond to different interplanar spacings, depending on whether the reduced or the oxidized form of the polymer is considered. The characteristic interplanar spacings for the reduced form are 0.95 , 0.47 , and $0.26 \mathrm{~nm}$ (Figure $8 \mathrm{a}$ ), and for the oxidized form they are $0.97,0.47,0.35$, and $0.26 \mathrm{~nm}$ (Figure $8 \mathrm{~b}$ ). Accordingly, the polymer coating structure exhibits a certain degree of crystallinity, in agreement with STM imaging.

3.5. Response of Polymer Electrodes to a Diffusion-Controlled Test Reaction. The potentiostatic current transients related to the

$$
\mathrm{Fe}(\mathrm{CN})_{6}{ }^{4-} \Rightarrow \mathrm{Fe}(\mathrm{CN})_{6}{ }^{3-}+\mathrm{e}^{-}
$$

reaction at the polymer electrodes were run at potentials in which the test reaction is under diffusion control. Thus, the polymercoated electrode was stepped from -0.4 to $0.6 \mathrm{~V}$ in order that reaction 2 proceeds at a constant potential. Prior to these experiments, blanks in $0.5 \mathrm{M} \mathrm{H}_{2} \mathrm{SO}_{4}$ were run to discriminate the proper polymer electrooxidation current components (peak $\mathbf{B}_{\mathrm{a}}$ and hump $\mathrm{C}_{\mathrm{a}}$ ). Current transient data after correction for the

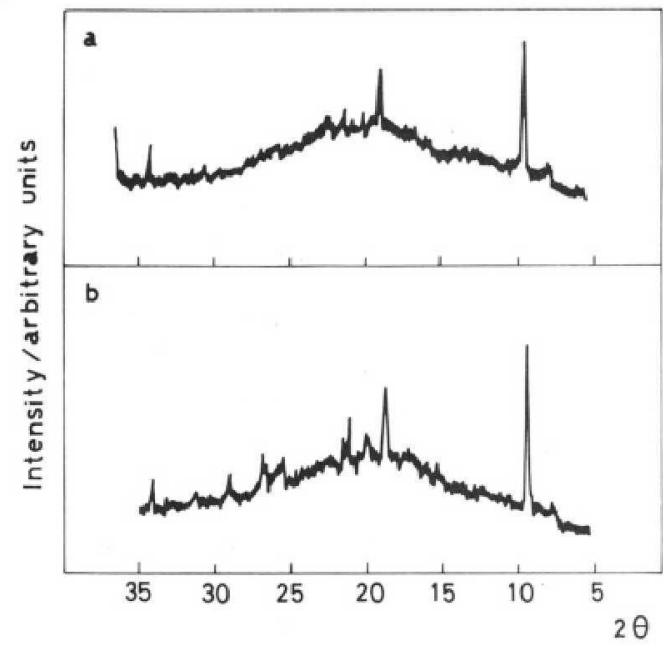

Figure 8. X-ray diffractograms of a polymer-coated Au electrode: (a) polymer coating reduced state; (b) polymer coating oxidized state.

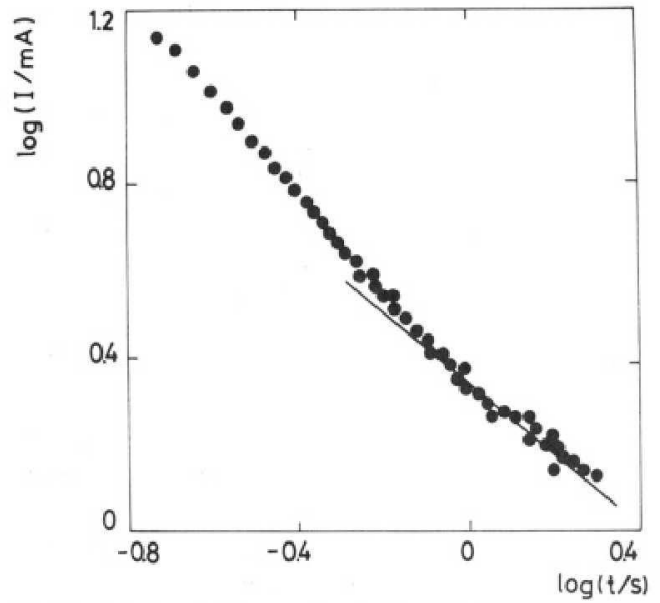

Figure 9. A $\log I v s \log t$ plot resulting for the $\left[\mathrm{Fe}(\mathrm{CN})_{6}\right]^{4-} \Rightarrow\left[\mathrm{Fe}(\mathrm{CN})_{6}\right]^{3-}$ $+\mathrm{e}^{-}$reaction in $0.5 \mathrm{M} \mathrm{H}_{2} \mathrm{SO}_{4}$ on a polymer coating Au electrode at 0.6 V. Experimental details are indicated in the text. The slope of the straight line is 0.8 .

blanks were displayed as $\log I$ vs $\log t$ plots (Figure 9). These plots show two linear regions which fulfill the following proportionality: ${ }^{12,20}$

$$
I \propto t^{-n}
$$

with $n \cong 1$ for $t<0.2 \mathrm{~s}$ and $n=0.80$ for $0.2<t<2 \mathrm{~s}$. Seemingly, data obtained for $t>2 \mathrm{~s}$ approach eq 3 with $n=0.5$.

\section{Discussion}

The structure of thin polymer coatings electrochemically grown on polyfaceted Au single crystal spheres is formed by nodular elements $(5 \mathrm{~nm}$ in minimum diameter and $15 \mathrm{~nm}$ in average diameter). These elements constitute chains which in the early stages of growth are aligned following the Au substrate topography. Conversely, the structure of relatively thick polymer coatings consists of nodular agglomerates which exhibit selfresemblance at different magnifications without any appreciable influence of Au substrate topography. The crystalline domains for thick polymer films revealed by $\mathrm{X}$-ray diffractometry could be confined to small nodular domains which may be composed of highly oriented polymer chains. ${ }^{21}$ The complete 3D delocalization of electron wave functions within the nodular domains means that substructural features (i.e., molecules) would certainly make imaging more difficult.

The topography of polymer coatings can be, in principle, related to the complex electrochemical kinetics of polymer growth as seen through anodic current transient data. 


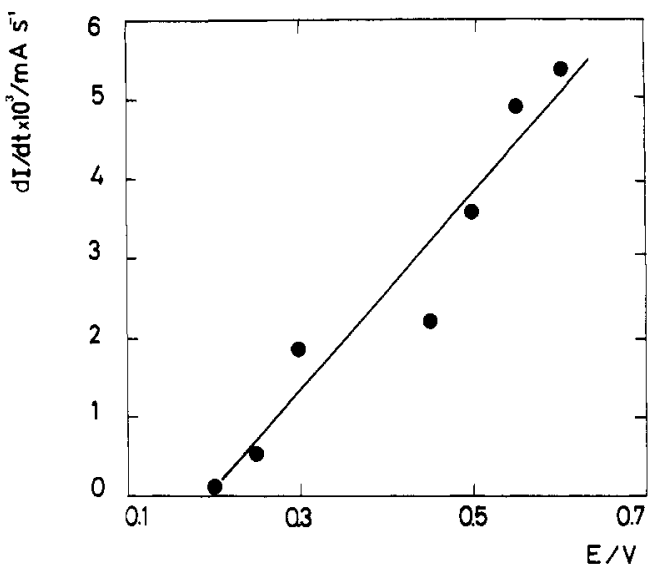

Figure 10. $\mathrm{d} l / \mathrm{d} t$ vs $E_{\mathrm{g}}$ plot at $25^{\circ} \mathrm{C}$.

4.1. A Possible Interpretation of Current Transients. Anodic current transients related to polymer coating formation show a current maximum appearing in the short time range (Figure 4a) followed by a linear current increase (Figure $4 b$ ). At the highest growth rate it appears that a limiting current value is attained. Besides, the first voltammogram exhibits a clear current loop in the anodic scan. These observations can be related to the contribution of nucleation and growth processes at the early electropolymerization stages.9.17 Thus, the nucleation stage of conducting polymers can be hindered by solution stirring as the critical concentration of soluble species required for the nucleation of the new phase cannot be reached. ${ }^{22}$ Conversely, no marked changes in the growth stage could be observed by stirring the solution. ${ }^{22}$ Then, it has been concluded that initially polymer growth is controlled by a surface reaction.9,22 These observations are consistent with the anodic current transient dependence on $E_{8}$ (Figure 4).

The linear current transient range can be explained considering the polymer formation as a progressive nucleation on substrate sites followed by the growth of independent linear chains under charge-transfer control. ${ }^{9}$ For this model $I$, the instantaneous current, is given by

$$
I=z F k\left(E_{\mathrm{g}}\right) A
$$

where $k\left(E_{\mathrm{g}}\right)$ is a potential-dependent growth rate constant and $A$ is the instantaneous overall polymer active area for polymer growth. For a progressive nucleation the following proportionality can be considered ${ }^{23}$

$$
A \propto A N_{0} \alpha t
$$

where $A$ is the capture area of the linear chain, $N_{0}$ is the number of nucleation sites at the substrate, and $\alpha$ is the site-to-nucleus conversion frequency. From eqs 4 and 5 the linear range dependence of $I$ and $t$ resulting from the experimental data (Figure 4) can be explained. Furthermore, the current transient slope vs $E_{\mathrm{g}}$ plot also fit a reasonable straight line, yielding a value of $R$ close to $300 \Omega$ (Figure 10). This figure reflects the influence of polymer resistance ${ }^{16}$ on the growth process.

The preceding analysis accounts for the initial stage of polymer layer growth, and it implies a continuous increase in the area of the growing interface. However, this is actually not the case as the polymer coating thickens; because the interface area related to the polymer layer growth attains saturation at large $\sum Q_{\mathbf{S N}}$ values (Figure 2). Hence, the progressive nucleation and growth model can explain the early stages of polymer growth when substrate sites are still available, in contrast to advanced stages of growth where the interface reaches a steady-state growth condition as $N_{0} \Rightarrow 0$; i.e., there are no sites available for nucleation at the substrate.
4.2. Surface Characterization. The thickening of polymer films with a nodular structure which develops roughness can be described in terms of the dynamic scaling theory. ${ }^{14}$ This approach provides information about the fractal properties and the growth mechanism of rough surfaces and can be applied to the analysis of rough metal surface STM images. ${ }^{10,24}$

Let us consider an object with an initial flat surface profile involving $N_{\mathrm{s}}$ growth sites in the length $L(t=0)$. As the object grows in a defined direction by material aggregation, the profile changes within the size $L$, and this change can be described by a single value function which depends on $L$ and $t$. For the surface width growing in the $i$ direction, the root-mean-square average roughness can be defined through the function $\xi^{i}(L, t)$ given by ${ }^{14}$

$$
\xi^{i}(L, t)=\left[1 / N_{\mathrm{s}} \sum_{j}\left[h_{j}-\mathbf{h}\right]^{2}\right]^{1 / 2}
$$

where $h_{j}$ is the object height measured along the $i$ direction at the $j$ position $(0 \leq j \leq L)$ and $\mathbf{h}$ is the average object height. Besides, $\xi$ and $L$ are related through the equation ${ }^{14}$

$$
\xi^{i}(L, t) \propto L^{\alpha} f(x)
$$

where $f(x)=\mathbf{h} / L^{\gamma}$. The function $f(x)$ has the following properties: $f(x)=$ constant for $x \Rightarrow \infty$, and $f(x)=x^{\alpha / \gamma}$ for $x \Rightarrow$ 0 . Thus, for either $\mathbf{h} \rightarrow 0$ or $t \rightarrow 0$, it results that

$$
\xi^{i}(\mathbf{h}) \propto \mathbf{h}^{\beta}
$$

After a certain critical thickness (time), $\xi^{l}$ reaches a steady state, and eq 8 becomes

$$
\xi^{i}(L) \propto L^{\alpha}
$$

The value of $D$, the local fractal dimension of the surface, can be obtained from the relationship ${ }^{14}$

$$
D=3-\alpha
$$

The above dynamic scaling concepts can be applied to STM data to obtain the value of $\alpha$ taking into account that $\xi^{l}=$ $\xi_{\text {stm }}^{i}$, where $\xi_{\text {stm }}^{i}$ is the mean-square average roughness determined by the STM scans in the $i$ direction $(i=x, y)$. Accordingly, rms values of the fluctuations of $h$ over each STM scan segment of length $L_{s}$ along the $x$ and $y$ directions can be determined after considering the standard plane correction in the calculations. ${ }^{10,25}$ More explicitly, in this work the following expressions have been used for $i=x: 10$

$$
\xi_{\mathrm{stm}}\left(L_{\mathrm{s}}\right) \propto\left[1 / N_{\mathrm{s}} \sum_{j}\left(h_{j}-h_{\mathrm{s}}\right)^{2}\right]^{1 / 2}
$$

and

$$
\xi_{\text {stm }} \propto L_{\mathrm{s}}^{\alpha}
$$

where $\mathbf{h}_{\mathbf{s}}$ is the average height of the surface profile of length $L_{s}$ in the $x$ direction. For each STM scan 250 pairs of points $\left(L_{s}, \xi_{\text {stm }}\right)$ have been obtained, $L_{\mathrm{s}}$ being varied from $S / 64$ to $S$, where $S$ stands for the total scan length. Finally, $\xi_{\text {stm }}$ represents the average value obtained from 256 scans of the same image for each $L_{\mathrm{s}}$ value.

First, the dynamic scaling was applied to STM images of the [111] pole region of a polyfaceted Au single crystal used as substrate for polymer coating (Figure 4 ). In this case, a $\log \xi_{\text {stm }}$ vs $\log L_{s}$ linear relationship with the slope $\alpha=0.85$ was obtained (Figure 11). As already reported from the analysis of computersimulated surfaces with known $\alpha$ values and similar file sizes $(256 \times 256)$, the dynamic scaling method applied to STM images requires a correction in the value of $\alpha$ for $\alpha>0.5 .24$ Accordingly, after correction, it results $\alpha \cong 1.0$. This figure shows a large degree of correlation of the surface topography at the [111] pole 


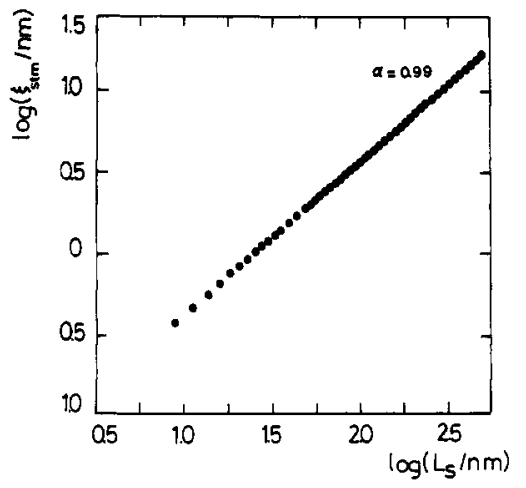

Figure 11. $\log \xi_{\operatorname{stm}} v s \log L_{\mathrm{s}}$ plot derived from the dynamic scaling-STM method. Data obtained from the image shown in Figure 5.

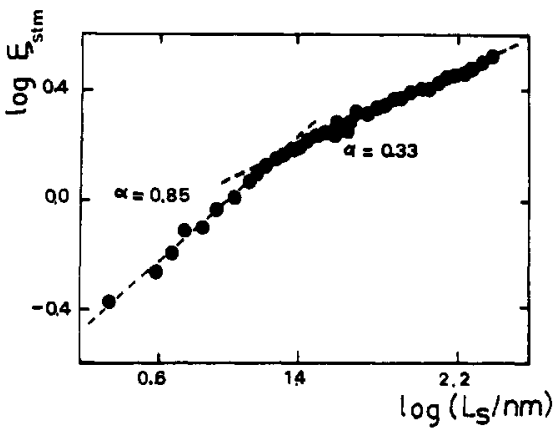

Figure 12. $\log \xi_{\text {stm }}$ vs $\log L_{\mathrm{s}}$ plot derived from the dynamic scaling-STM method. Data obtained from the image shown in Figure $7\left(Q_{\mathrm{aN}}=22\right.$ C, $\left.E_{\mathrm{B}}=0.35 \mathrm{~V}\right)$.

region of the polyfaceted Au single crystal. From eq 7 it results $D \cong 2.00 \pm 0.05$, as it should be expected for a stepped surface.

Otherwise, the extension of the dynamic scaling analysis to STM images of a polymer-coated electrode surface at advanced stages of growth $\left(t \Rightarrow \infty, Q_{\mathrm{a} N}=22 \mathrm{C}\right)$ leads to $\log \xi_{\mathrm{stm}}$ vs $\log L_{\mathrm{s}}$ plots with two linear regions (I and II) with slopes $\alpha(\mathrm{I})=0.70$ \pm 0.05 for $L_{\mathrm{s}}<15 \mathrm{~nm}$ and $\alpha(\mathrm{II})=0.33 \pm 0.05$ for $L_{\mathrm{s}}>15 \mathrm{~nm}$ (Figure 12). Accordingly, averaged values from 20 images after correction are $\alpha(\mathrm{I})=0.85 \pm 0.05$ for $L_{s}<15 \mathrm{~nm}$ and $\alpha(\mathrm{II})=$ $0.33 \pm 0.05$ for $L_{\mathrm{s}}>10 \mathrm{~nm}$. It should be noted that the transition in the value of $\alpha$ occurs for $L_{\mathrm{s}} \cong 15 \mathrm{~nm}$, a figure which is close to the average size of the nodular elements forming the polymer structure. Then, this analysis indicates that the surface of nodular elements approaches apparently the behavior of an Euclidean surface as $D(I)=2.15 \pm 0.05$, whereas the surface of agglomerates formed by aggregation of nodular elements behaves as a selfaffine fractal with $D(I I)=2.66 \pm 0.05$. The apparent Euclidean behavior of nodular elements is consistent with the complete $3 \mathrm{D}$ delocalization of electron wave functions of the ordered polymer chains within nodules. 20

The value of the kinetic roughening exponent $\beta$ can be estimated from eq 9 considering that $Q_{S N} \propto \xi$ and $\sum Q_{S N} \propto$ h. These relationships imply that $Q_{S N}$ is proportional to the polymer surface, and $\sum Q_{\mathrm{S} N}$ is proportional to the nonfractal polymer mass, i.e., $\sum Q_{\mathrm{S} N} \propto \mathbf{h}^{13}$ The first assumption is sustained by the fact that monomer electrooxidation behaves as a surface-controlled reaction as it can be inferred from the current transient dependence on $E_{\mathrm{g}}$ (Figure 4). Therefore, a loss of surface area for monomer electrooxidation due to the presence of surface irregularities should be discarded. The second assumption can be justified on the basis of previous observations that the polymer structure seems to be space filling. ${ }^{13}$ It should also be noted that these relationships were obtained at the early stages of growth so that the condition $t=0$ in eq 9 is fulfilled. Accordingly, the value of $\beta$ can be derived from the $\log Q_{\mathrm{SN}}$ vs $\log \sum Q_{\mathrm{SN}}$ plot. Data resulting from Figures 1 and 2 furnishes a good linear plot with the slope $\beta \cong$ 0.73 (Figure 13).

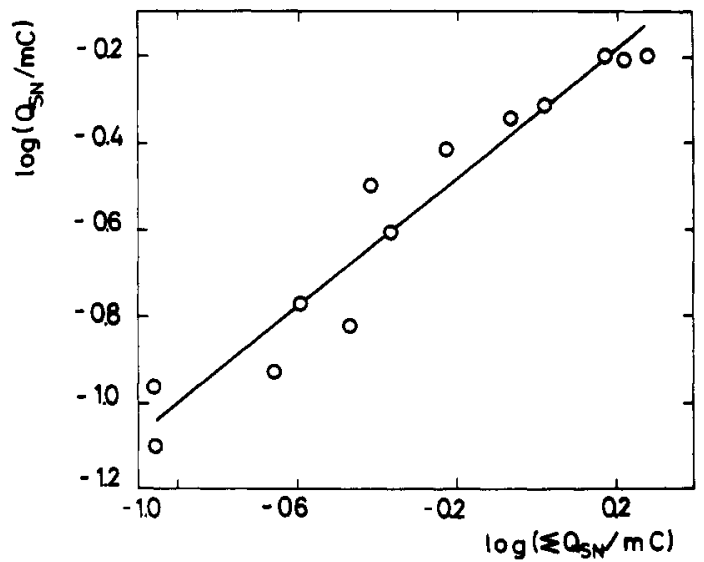

Figure 13. $\log Q_{s}$ vs $\log \sum_{N} Q_{s N}$ plot. Data taken from Figure 2.

It has been demonstrated that the exponents $\alpha$ and $\beta$ must satisfy in all dimensions the following relationship ${ }^{14}$

$$
\alpha+\alpha / \beta=2
$$

Equation 13 indicates that the value $\beta=0.73$ should be related to the value $\alpha=0.85$ obtained from $L_{\mathrm{s}}<15 \mathrm{~nm}$. This is consistent with the electrooxidation of monomers of about $1 \mathrm{~nm}$ in average size at the surface of nodular elements $\left(L_{\mathrm{s}}<10 \mathrm{~nm}\right)$ which becomes independent of the rough topography of larger agglomerates. Therefore, the surface properties of polymer coatings for $L_{\mathrm{s}}<10$ $\mathrm{nm}$ closely fulfill eq 8 as $\alpha(\mathrm{I})=0.85$ and $\beta(\mathrm{I})=0.73$.

4.3. Electrochemical Behavior of Polymer-Coated Electrodes. The preceding discussion can be extended to the interpretation of the different values of $n$ resulting from the test reaction data on polymer-coated electrodes (Figure 9). It is known that, for a diffusion-controlled electrochemical reaction involving soluble species in solution, $n$ and $D$ are related by the equation ${ }^{21}$

$$
n=(D-1) / 2
$$

Despite the complexity of the electrochemical reaction 2 at the polymer-coated electrode, experimental data were plotted as log $I$ vs $\log t$ (Figure 9). This plot exhibits a first linear portion with the slope $n \cong 1$ for $t<0.2 \mathrm{~s}$, which agrees with that resulting from the blank, and it can be explained by considering that the test reaction at surface domains characterized by $L_{s}<10 \mathrm{~nm}$ is controlled by the own polymer electrooxidation reaction. Otherwise, for $0.2<t<2 \mathrm{~s}$, results fit a straight line with the slope corresponding to $n=0.80$, a figure which is consistent, within the experimental error, with $\alpha(\mathrm{II}) \cong 0.33$ and $D(\mathrm{II}) \cong 2.66$, as derived from the dynamic scaling-STM analysis for $L_{s}>10 \mathrm{~nm}$. This means that as far as the diffusion current is concerned, for $t>0.2 \mathrm{~s}$, the corresponding diffusion layer thickness scales the large roughness caused by those large agglomerates which dominate the polymer-coated electrode surface at $L_{\mathrm{s}}>15 \mathrm{~nm}$. Finally, for $t>2 \mathrm{~s}$, the $\log I$ vs $\log t$ plot approaches the conventional slope $n=0.5$; i.e., when the diffusional layer thickness becomes larger than the size of the largest irregularities, the electrode surface behaves as a smooth surface.

4.4. Polymer Surface Characteristics and the Likely Polymer Growth Mechanisms. To account for the kinetics of the conducting polymer growth on a substrate with the above-described topography, a relatively simple electropolymerization mechanism is proposed. This mechanism can be simulated by the Monte Carlo method considering (i) the starting substrate surface (SS) and the monomer units, (ii) the transport of monomers to the SS, (iii) monomer electrooxidation, and (iv) the incorporation of electrooxidized species into polymer growth sites. The SS is a cross section (2D) of an ideally flat metal surface in contact with monomers in the solution. Both SS and monomers in the solution are projected on the square lattice. Typical dimensions of the 2D 
domain are 100 substrate particles per line and 80 or 60 lines describing monomers in the solution. Monomer interaction terms have been disregarded in this approach. The transfer of monomers from the solution to SS involves a random walk. ${ }^{26}$ The monomers in the solution are able to move at random with a motion probability $P_{\mathrm{m}}=0.25$ for each $(x,-x ; \leftrightarrow y,-y)$ directions on the plane. During a Monte Carlo time unit all monomers are moved once on average. A complete description of the simulation procedure is given elsewhere. ${ }^{27}$

Monomers which arrive at SS may react according to one of the following pathways. In pathway $\mathrm{i}$, monomers can be electrooxidized at SS with the probability $P_{\text {ox }}$ yielding a radical species. This species can diffuse outward or remain at the SS. The formation of soluble monomer and/or oligomer electrooxidized forms has been inferred from rotating disk electrode data. ${ }^{22}$ In pathway ii, monomers can be electrooxidized with the same probability $P_{0 x}$ yielding radicals at top sites of oxidized chains bonded to SS. Radicals formed through pathways i and ii can move at random in the solution until they are incorporated either into the end of a chain with a probability $P_{\mathrm{e}}$ or laterally with a probability $P_{1}$. In the calculation $P_{\mathrm{e}} \gg P_{1}$ was used; i.e., the sticking probability at the chain ends was always greater than the lateral sticking probability, to assure a preferential linear growth and low branching. Profiles of the snapshots (Figure 14) generated with this model and the set of parameters given in the figure's caption imply that $P_{0 x}>P_{\mathrm{e}} \gg P_{1}$. Under these conditions the rate-determining step in polymer growth mechanism is the radical incorporation into the surface growth site. The snapshots also show that radicals lie close to all surface growing sites so that these sites attain the same growth probability. Therefore, the kinetics of the overall process is consistent with a rate-determining surface reaction. One should note that the structure generated throughout the preceding model displays a porous structure with a uniform density, i.e., a nonfractal mass and a rough profile. The dynamic scaling of surface is consistent with a self-affine surface with $\alpha=0.44$ and $\beta=0.33$. These values are close to those predicted by the $2 \mathrm{D}$ computer simulation of the Eden model. ${ }^{28,29}$

Monte Carlo calculation was also extended for $P_{\text {ox }}<P_{\mathrm{e}}$. Then, 2D profiles correspond to open structures with deep crevices depleted of radical species. Under these conditions the growth process is controlled by a Laplacian field ${ }^{26}$ rather than by a surface reaction.

In conclusion, all of the above-mentioned features of the polymer coating growth mechanism for $P_{\mathrm{ox}}>P_{\mathrm{e}}$ retain the most relevant characteristics of the Eden model. It should be noted that when the Eden model is simulated in 3D it results in $\alpha=0.33$, a figure which coincides with $\alpha=0.33$ derived from the STM images of the polymer-coated electrode by the dynamic scaling (Figure 12). In principle, the smallest irregularities in the snapshots resulting from Monte Carlo simulation are the depositing particles themselves, so that in the polymer coating the smallest irregularities should correspond to either monomers or small oligomers formed in the course of the electrooxidation process. Conversely, STM imaging information shows that the smallest irregularities are due to polymer nodules. Presumably, chain-chain interactions might be involved in the formation of these nodular elements, so that this type of interactions would provide an explanation for the appearance of a cutoff associated with the the self-affine fractal range of the polymer surface. In fact, this type of interaction should be expected at the sol/gel transition which usually takes place along polymerization reactions. ${ }^{30}$

\section{Conclusions}

The polymer structure depends on the coating thickness. At the early stages of growth the polymer structure reflects the substrate topography, whereas at advanced stages of growth it evolves to a topography dominated by agglomerates made of small nodular elements. a

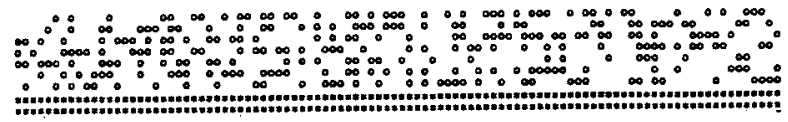

b
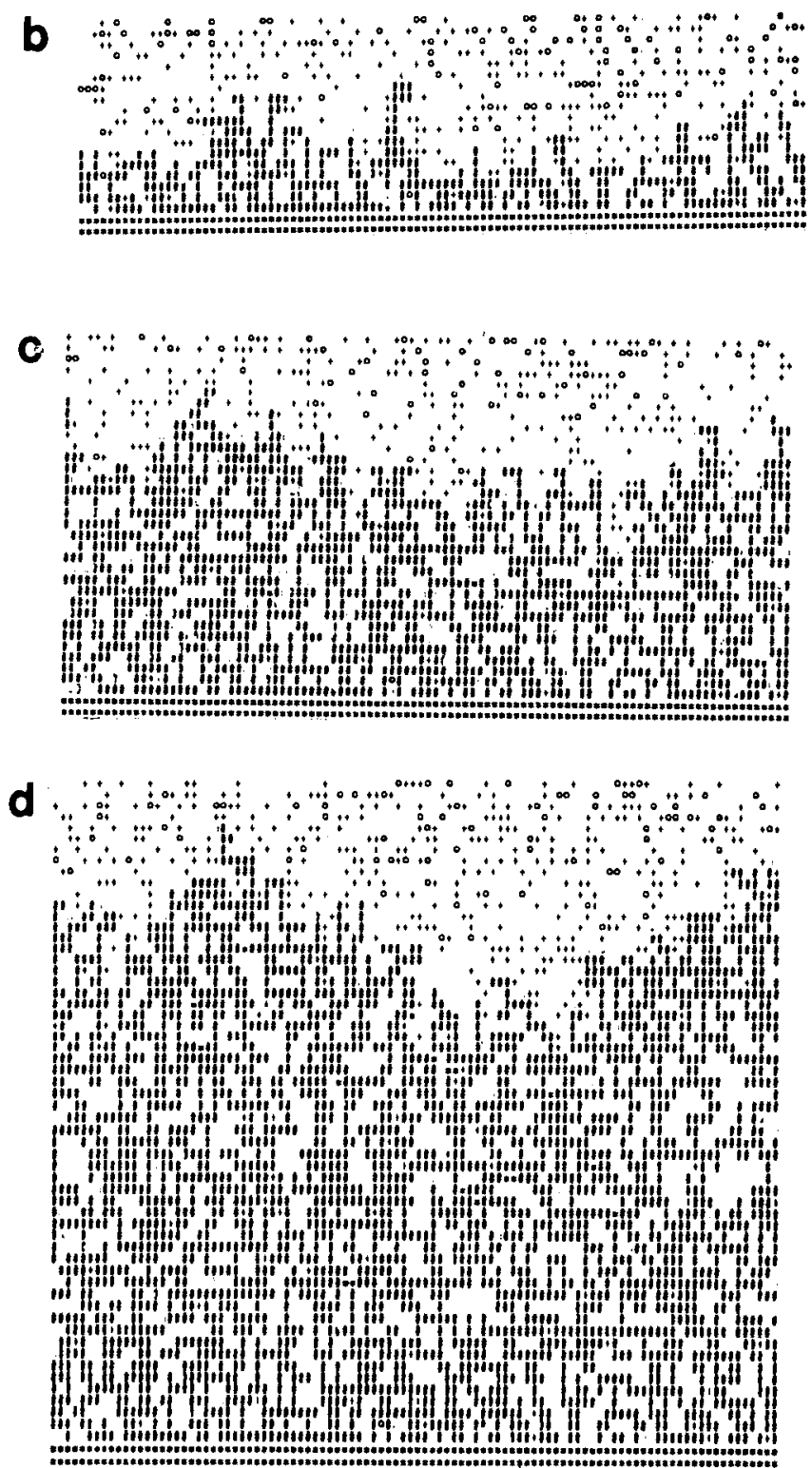

Figure 14. Snapshots corresponding to Monte Carlo simulations of polymer coating growth at different times (in Monte Carlo time units) considering $P_{0 \mathrm{x}}=1, P_{\mathrm{e}}=0.01$, and $P_{1}=0.001$. (a) $t=0$, (b) $t=40$, (c) $t=100$, and (d) $t=160$. Substrate $\left({ }^{*}\right)$, monomers $(0)$, oxidized units in solution (+), and polymer phase (\#) are indicated.

At advanced stages of growth the coating topography exhibits self-resemblance and can be described as a self-affine fractal surface with $\alpha \cong 0.85$ and $\beta \cong 0.73$ for scaling lengths smaller than the average nodular element diameter and $\alpha \cong 0.33$ for larger scaling lengths.

The value $\alpha \cong 0.33$ coincides with that obtained from Monte Carlo simulations of a simple polymer growth mechanism controlled by a surface reaction. However, the cutoff length, which is related to the nodular average size, cannot be entirely explained by a simple aggregation model of monomers or small oligomers controlled by surface reactions. Chain-chain interactions could explain the development of the small ordered domains.

The surface reaction control of the electropolymerization implies that monomers or small oligomers probe all polymer active surface sites. Accordingly, the reaction occurs at small nodular elements, and it is far from large scale surface roughness influence. 
For the $\mathrm{Fe}(\mathrm{CN})_{6}{ }^{4-} \Rightarrow \mathrm{Fe}(\mathrm{CN})_{6}{ }^{3-}+\mathrm{e}^{-}$reaction the diffusion layer thickness probes the large scale surface roughness, as a $\log$ (diffusional current) vs $\log$ (time) plot exhibits a certain linear range with an anomalous slope $n$, which is compatible with $\alpha=$ 0.33 .

It is possible to link the electrode topography and the electrochemical reaction kinetics even for strongly disordered surfaces such as that formed by $o$-toluidine polymer coatings on a metal substrate.

Acknowledgment. Financial support from the Consejo Nacional de Investigaciones Científicas y Técnicas (CONICET) of Argentina is acknowledged.

\section{References and Notes}

(1) Dlaz, A. F.; Bargon, J. In Handbook of Conducting Polymers; Skotheim, A., Ed.; Marcel Dekker: New York, 1986; Vol. 1, p 81.

(2) Warren, L. F.; Walker, J. A.; Anderson, D. P.; Rhodes, C.; Buckley, L. J. J. Electrochem. Soc. 1989, 136, 2286. 181.

(3) Genies, E. M.; Lipkowski, M.; Tsintavis, C. New J. Chem. 1988, 12,

(4) Huang, W. S.; Humphrey, B. D.; McDiarmid, A. G. J. Chem. Soc., Faraday Trans. 1986, $1,82$.

(5) Yang, R.; Naoi, K.; Evans, D. E.; Smyrl, W. A.; Hendrickson, W. A. Langmuir $1991,7,556$.

(6) Porter, T. L. Surf. Sci. 1993, 293, 81.

(7) Yang, R.; Dalsin, K. M.; Evans, D.; Christensen, D. F.; Hendrickson, W. A. J. Phys. Chem. 1989, 93,511 and references therein.

(8) Aoki, K. J. Electroanal. Chem. 1991, 300, 13 2249 .

(9) Bode, K.; Tsakova, V.; Schultze, J. W. Electrochim. Acta 1992, 37,

(10) Salvarezza, R. C.; Vázquez, L.; Ocón, P.; Herrasti, P.; Vara, J. M.; Arvia, A. J. Europhys. Lett. 1992, 20, 727.
(11) Gómez-Rodríguez, J. M.; Vázquez, L.; Baró, A.; Salvarezza, R. C.; Vara, J. M.; Arvia, A. J. J. Phys. Chem. 1992, 96, 347.

(12) Ocón, P.; Herrasti, P.; Vázquez, L.; Salvarezza, R. C.; Vara, J. M.; Arvia, A. J. J. Electroanal. Chem. 1991, 319, 101.

(13) Villeret, B.; Nechtschein, N. Solid State Commun. 1987, 64, 435.

(14) Family, F. Physica $A$ 1990, 168, 561 and references therein.

(15) Canullo, J. C.; Triaca, W. E.; Arvia, A. J. J. Electroanal. Chem. 1986, 200, 397.

(16) Ocón, P.; Herrasti, P. New J. Chem. 1992, 16, 501.

(17) Asavapiriyanont, S.; Chandler, G. K.; Gunawardena, G. A.; Pletcher, D. J. Electroanal. Chem. 1984, 177, 229.

(18) Sommerfeld, D. A.; Cambron, R.; Beebe, Jr., T. J. Phys. Chem. $1990,94,8926$.

(19) Porter, T. L.; Oden, P. I.; Lee, C. Y.; Caple, G. J. Vac. Sci. Technol. A 1992, 10, 606.

(20) Wang, Z. H.; Li, C.; Scherr, E. M.; MacDiarmid, A. G.; Epstein, A. J. Phys. Rev. Lett. 1991, 66, 1745.

(21) Pajkossy, T.; Nyikos, L. Electrochim. Acta 1989, 34, 171.

(22) Fermin, D.; Garcia-Pastoriza, E.; Scharifker, B. R. Abstracts 43rd ISE Meéting, Córdoba, Argentina, 1992; p 154.

(23) Hernández Creus, A.; Carro, P.; González, S.; Salvarezza, R. C.; Arvia, A. J. Electrochim. Acta 1992, 37, 2215.

(24) Vázquez, L.; Salvarezza, R. C.; Herrasti, P.; Ocón, P.; Vara, J. M.; Arvia, A. J. Appl. Surf. Sci. 1993, 70-71, 413.

(25) Krim, J.; Hevaert, I.; Haesendock, C. H.; Bruynseraede, I. Phys. Rev. Lett. 1993, 70, 57.

(26) Witten, T. A.; Sander, L. M. Phys. Rev. Lett. 1981, 47, 1400.

(27) Salvarezza, R. C.; Alonso, C.; Vara, J. M.; Albano, E.; Martin, H. O.; Arvia, A. J. Phys. Rev. B 1990, 41, 12502.

(28) Eden, M. In Proceedings of the 4th Symposium on Mathematical Statistics and Probability; Feyman, J., Ed.; University of California Press: Berkeley, 1961; Vol. 4, p 223.

(29) Vicsek, T. Fractal Growth Phenomena; World Scientific: Singapore, 1989.

(30) Flory, P. J. Principles of Polymer Chemistry; Cornell University Press: Ithaca, 1953. 